\title{
ENSAYO DE APROXIMACION A LAS CUESTIONES PLANTEADAS POR LA CRISIS SANITARIA EN RELACIÓN CON EL ESTADO AUTONÓMICO
}

\author{
Francisco Javier Matia Portilla \\ Catedrático de Derecho Constitucional, \\ Universidad de Valladolid
}

Cómo citar este artículo / Citation: Matia Portilla, F. J. (2021). Ensayo de aproximación a las cuestiones planteadas por la crisis sanitaria en relación con el Estado Autonómico.

Tudela Aranda, J. (coord.)

Estado Autonómico y covid-19,

Colección Obras colectivas, Fundación Manuel Giménez Abad, Zaragoza.

DOI: https://doi.org/10.47919/FMGA.OC21.0209

SUMARIO: I. INTRODUCCIÓN - II. UNA VALORACIÓN GLOBAL DE LA RESPUESTA DEL ESTADO AUTONÓMICO A LAS EXIGENCIAS DE LA CRISIS - III. UNA APROXIMACIÓN A LOS PROBLEMAS PLANTEADOS POR LOS DOS MODELOS DE ESTADO DE ALARMA - IV. LÍMITES DE LA CAPACIDAD DE LAS COMUNIDADES AUTÓNOMAS PARA INCIDIR SOBRE DERECHOS $Y$ COMPETENCIAS - V. VALORACIÓN DE LAS RELACIONES INTERGUBERNAMENTALES DURANTE LA PANDEMIA - VI. LECTURAS PARA EL FUTURO 
fundación

Manuel Giménez Abad

de Estudios Parlamentarios ydel Estado Autonómico

\section{INTRODUCCIÓN}

La pandemia causada por la covid-19 está provocando profundas consecuencias transversales ${ }^{1}$. Si bien es muy pronto para tener un balance adecuado sobre las mismas, es conveniente iniciar una reflexión que deberá prolongarse en el tiempo. Parece evidente que, además de posibles errores en la gestión por unas y otras autoridades, se han puesto de manifiesto deficiencias estructurales en distintos ámbitos de nuestro Estado.

Uno de los focos de atención ha sido la organización territorial del poder. El modelo comúnmente denominado Estado autonómico se ha convertido en uno de los protagonistas del debate. Entre todas las cuestiones suscitadas, dos han tenido particular incidencia pública. Por un lado, el rol que corresponde al Gobierno de la Nación en una crisis que excede los presupuestos ordinarios de la gestión de la sanidad. Por otro, el equilibrio entre la bondad y necesidad de la diversidad de respuestas que permite la descentralización y los equívocos que puede suscitar una heterogeneidad no coordinada.

Es pronto para afirmar si el modelo representado por el Estado autonómico sale debilitado o fortalecido de la crisis. Una mirada comparada nos enseña que la eficacia en la respuesta a la crisis no ha dependido de la forma de organización territorial del poder. En nuestro propio modelo, se pueden observar pareceres diversos. Así, merecerían un juicio positivo la capacidad para flexibilizar las respuestas en función de las circunstancias de cada territorio o la emergencia de relevantes niveles de coordinación entre las Comunidades Autónomas si bien, la mayoría de las veces, de manera informal. Pero también se han podido observar hechos que merecen ser ponderados para ser corregidos. Entre ellos, se puede citar la debilidad de las Comunidades para poder responder a ciertas situaciones; un exceso de heterogeneidad en las respuestas que ha provocado confusión entre los ciudadanos; o la existencia de dos modelos totalmente contrapuestos de gestión por parte del Estado. Junto a ellos se encuentra la relevancia del debate

${ }^{1}$ Contribución finalizada el 13 de febrero de 2021, fecha en que todos los hipervínculos citados en las siguientes notas se encuentran activos y en funcionamiento. 
fundación

Manuel Giménez Abad

deEstudiosParlamentariosydelEstado Autonómico

suscitado sobre la ortodoxia jurídica de algunas de las decisiones adoptadas bien por el Estado bien por las Comunidades Autónomas.

Así, durante estos meses se han planteado numerosas cuestiones de gran relevancia jurídica. Debatirlas y, al menos, aproximarnos a algunas conclusiones fue el objetivo de la convocatoria de este año 2020 que anualmente hacemos de forma conjunta con la Fundación Adenauer en el Monasterio de Piedra.

\section{UNA VALORACIÓN GLOBAL DE LA RESPUESTA DEL ESTADO AUTONÓMICO A LAS EXIGENCIAS DE LA CRISIS}

Antes de realizar una valoración, subjetiva y provisional sobre la pregunta planteada, resulta conveniente suscitar una previa, y es si resulta conveniente que se afronte una pandemia desde las administraciones regionales o sería más lógico que fuera abordada por el Gobierno de la Nación. Si se acepta la hipótesis de que para afrontar esta crisis resulta preciso la adopción de un estado de emergencia constitucional (no entraremos ahora en si debe optarse por alarma o excepción) resulta claro que el protagonismo debe corresponder al Gobierno del Reino de España, sometido al control parlamentario del Congreso de los Diputados.

A nuestro entender, resulta evidente que ante una crisis sanitaria global no es comprensible que haya diecisiete centros de decisión, y el hecho de que exista una autoridad central responsable (para adoptar las decisiones y para someterse al escrutinio del Congreso de los Diputados) no impone, obviamente, que se adopten medidas idénticas para los distintos territorios (no solamente regionales; también locales y provinciales). El Gobierno puede decretar medidas diferenciadas para los distintos territorios en función de los datos sanitarios, y puede involucrar a los Gobiernos regionales, tanto a la hora de conocer la realidad asistencial (fase ascendente) como en la ejecución de las decisiones adoptadas (fase descendente). Es más cuestionable que sean esos gobiernos los que adopten las decisiones. Lo es, fundamentalmente, porque el control del Congreso de los 
Diputados es esencialmente insuficiente y limitado $^{2}$, ya que se reduce al marco configurado por el Real Decreto de Alarma, y porque la actuación de los Gobiernos autonómicos se impone sin problemas sobre las Asambleas Regionales.

En todo caso, como es sabido, y resulta jurídicamente discutible, el Gobierno ha optado por establecer un marco de actuación en el Real Decreto 926/2020, de 25 de octubre, por el que se declara el estado de alarma para contener la propagación de infecciones causadas por el SARS-CoV-2, permitiendo a los Presidentes autonómicos que, como autoridades competentes delegadas, puedan modularlas.

A partir de este dato, cobra sentido la cuestión ¿ha sido adecuada la respuesta del Estado autonómico a la pandemia? Y la contestación que debe darse es negativa.

Una buena parte de las razones que justifican esta respuesta tienen su origen en la naturaleza de la pandemia. Ya se ha indicado que estamos ante un problema estatal que merecía una respuesta centralizada. Así ocurrió, de hecho, con el primer estado de alarma, en el que se impusieron unas medidas generales. Ya se ha adelantado, y debe insistirse en ello, que el hecho de que exista un nivel central de decisión no implica, necesariamente, que el Gobierno no hubiera podido establecer una gradación de escenarios que permitiera la adaptación de las restricciones a distintos territorios, dependiendo de la situación de cada uno de ellos.

Pero también existen argumentos que permiten responder negativamente a la cuestión planteada a partir del análisis de la actuación de las propias Comunidades Autónomas. En la primera fase de decisiones centralizadas, algunas Comunidades Autónomas ( $\mathrm{y}$, también, el Gobierno de la Nación) se han servido de la pandemia para cuestionar a las Comunidades Autónomas gobernadas por otros

2 Conviene recordar, a mayor abundamiento, que en 2020 se han tramitado once leyes (de las que todas, menos la de Presupuestos, han sido aprobado, en primera instancia, por comisiones parlamentarias y que muchas de ellas se han tramitado, además por el procedimiento de urgencia). También se han aprobado tres leyes orgánicas. Estas cifras contrastan con la emisión de treinta y nueve decretos-leyes. Al amparo de estos datos, podemos concluir que también el Parlamento ha sido minusvalorado en su función legislativa. 
fundación

Manuel Giménez Abad

deEstudios Parlamentarios delEstado Autonómico

partidos. Además, y en segundo lugar, también en esa fase han entrado en una competición para conseguir y acaparar material sanitario, en contra del interés general que requería un reparto nacional, racional y solidario de los (escasos) recursos existentes.

Esta situación ha empeorado y se ha agravado significativamente tras el primer estado de alarma y, de forma especial, durante el que vivimos ahora. Siendo cierto que las Comunidades Autónomas disponían de sus competencias sanitarias, como ahora las tienen en el marco del vigente estado de alarma, bien podían haber tratado de alcanzar acuerdos con otras Comunidades Autónomas, que posibilitarán que los ciudadanos pudieran tener una mínima planificación sobre sus vidas y sus negocios. Nada de esto ha ocurrido. No es solamente que cada Comunidad Autónoma haya decidido de forma unilateral sus medidas; es que, además, las han variado de semana en semana, provocando estupor en las personas que ya no saben qué se puede hacer y qué no se puede hacer en cada momento. Es de justicia señalar que estos fenómenos (ejercicio de la competencia regional al límite e incapacidad ontológica para alcanzar acuerdos) no son novedosos, sino que encierran una de las mayores fallas de nuestro modelo de Estado.

Un ejemplo del despropósito vivido lo acredita la decisión del Gobierno de la Nación de permitir el encuentro con allegados (rectius, libre circulación) durante las navidades, permitiendo, eso sí, a las Comunidades Autónomas que pudieran restringir la movilidad. El Estado central, ante una situación que se sabía que iba a generar un serio rebrote de la enfermedad que se traduciría en muchas muertes y en un nuevo colapso sanitario, se abstiene de su deber, para que sean las regiones las que asuman el coste político derivado de las restricciones. A partir de aquí cada una hace, literalmente, lo que quiere, desde impedir el acceso de los no residentes (Comunidad Valenciana) hasta no imponer límite alguno (Cantabria). Y hemos visto en fechas más recientes cómo, mientras el Gobierno de la Nación se resiste a tomar medidas ante unos índices muy cercanos a los que justificaron el confinamiento para permitir así que se celebren en febrero las elecciones autonómicas catalanas, el Gobierno de la Generalitat apela ahora a la salud 
pública para retrasarlas. Somos conscientes de que lo único que preocupa a uno y otro son sus intereses electorales.

En resumen, lo razonable habría sido que el centro de decisión en una pandemia fuera único y atendiera al interés general, contando con las administraciones territoriales (en sentido amplio) para conocer la situación y, en su caso, para ejecutar o implementar las medidas adoptadas.

\section{UNA APROXIMACIÓN A LOS PROBLEMAS PLANTEADOS POR LOS DOS MODELOS DE ESTADO DE ALARMA}

El Gobierno de la Nación ha dictado dos estados de alarma consecutivos para todo el territorio a través de los Reales Decretos 463/2020, de 14 de marzo, que cesó su vigencia el 21 de junio3; y 926/2020, de 25 de octubre, aún en vigor ${ }^{4}$. Además, dictó otro estado de alarma aplicable exclusivamente a algunos municipios madrileños 5 .

El primer Real Decreto opta por una utilización canónica o clásica del estado de alarma, estableciendo como autoridad competente al Gobierno de España, y adoptando unas medidas restrictivas comunes para todo el Estado, que se fueron matizando a lo largo del tiempo posteriormente.

\footnotetext{
${ }^{3}$ Ver los Reales Decretos 476/2020, de 27 de marzo (añade la Disposición Adicional 6), 487/2020, de 10 de abril; 492/2020, de 24 de abril (modifica los arts. 7 y 10.6); 514/2020, de 8 de mayo (añade la Disposición Adicional 7); 537/2020, de 22 de mayo (deroga las disposiciones adicionales 2 a 4); 555/2020, de 5 de junio, que se aprueban tras los acuerdos de autorización del Congreso para la prórroga del estado de Alarma que se vehicula a través de Resoluciones de 25 de marzo, 9 de abril, 22 de abril, 6 de mayo, 20 de mayo y 3 de junio; (efectos 7 a 21 de junio). El Tribunal Constitucional ha admitido a trámite el recurso de inconstitucionalidad interpuesto por más de cincuenta diputados contra el Real Decreto 463/2020 (y los 465, 476, 487 y 492/2020) por eventual vulneración de los arts. 10.1, 16, 17, 19, 21, 25, 27, 35, 38, 55 y 116 CE.

${ }^{44}$ Mediante Resolución de 29 de octubre de 2020 del Congreso de los Diputados, se ordena la publicación del Acuerdo de autorización de la prórroga del estado de alarma declarado por el Real Decreto 926/2020, que llegará, si lo estima preciso el Gobierno, hasta el 9 de mayo de 2021. Se dicta entonces el Real Decreto 956/2020, de 3 de noviembre, que modifica los arts. 9, 10 y 14, y añade una disposición adicional Segunda.

${ }^{5}$ A través del Real Decreto 900/2020, de 9 de octubre, por el que se declara el estado de alarma para responder ante situaciones de especial riesgo por transmisión no controlada de infecciones causadas por el SARS-CoV-2.
} 
Aunque se ha respetado escrupulosamente la obtención de las pertinentes autorizaciones del Congreso de los Diputados para su prórroga, lo cierto es que el control parlamentario sobre la actuación del Gobierno ha sido limitado. Es de justicia hacer notar que la propia pandemia, en su fase más álgida, hacía complicado reunir a los Plenos de las Cámaras (no solamente en el ámbito estatal, también en el autonómico). Se ha producido, lógicamente, un cierto impasse mientras que los parlamentos retomaban su actividad, y esta se ha visto seriamente afectada por las circunstancias ${ }^{6}$. En lo que atañe al Congreso de los Diputados, que es lo que ahora nos interesa, recordamos, gracias a Piedad García-Escudero Márquez ${ }^{7}$, un resumen de su actuación: (1) el 12 de marzo (dos días antes de la declaración del estado de alarma) la Presidenta suspende la actividad parlamentaria durante dos semanas ${ }^{8}$; (2) el 18 de marzo comparece el Presidente del Gobierno ante el Pleno, al amparo del artículo 165 RCD, con la asistencia presencial de una veintena de Diputados; (3) el 25 de marzo se celebra un Pleno (al que asisten 49 parlamentarios, pudiendo utilizar el resto el voto telemático), en el que se acuerda la primera prórroga del estado de alarma, a la que seguirán otras ${ }^{9}$; (5) el 15 de abril se produce la primera sesión plenaria de

\footnotetext{
${ }^{6}$ Pueden consultarse, en relación con las asambleas legislativas de las Comunidades Autónomas, el volumen colectivo El Parlamento ante la Covid-19. Fundación Manuel Giménez Abad. Zaragoza, 2020, disponible en

https://www.fundacionmgimenezabad.es/sites/default/files/Publicar/publicaciones/documentos/mon ografia_8_junio_2020_1.pdf. y, en el plano comparado, Díaz Crego, María \& Mańko, Rafał: "Parliaments in emergency mode: How Member States' parliaments are continuing with business during the pandemic". Briefing European Parliament, disponible en https://www.europarl.europa.eu/RegData/etudes/BRIE/2020/649396/EPRS_BRI(2020)649396_EN. pdf.

7 "Actividad y funcionamiento de las Cortes Generales durante el estado de alarma por Covid-19". En VV.AA. El Parlamento ante..., cit., pp. 20-23.

8 Víctor Ernesto Alonso Prada considera que la declaración del estado de alarma hace decaer esta suspensión, por diversas razones (entre las que se incluye el tenor literal del artículo 116.5 CE). En "El control del Congreso de los Diputados al Gobierno y la actividad parlamentaria durante el estado de alarma”. En Revista del Gabinete Jurídico de Castilla-La Mancha 21 (2020), pp. 75 y ss. ${ }^{9}$ En esta primera ocasión se añade una disposición adicional sexta al documento presentado por el Gobierno, a instancia del Grupo Vasco, y se convalidan cinco Decretos-leyes. En esta línea se inscriben los Plenos de los días 9 de abril, que convalida tres nuevos Decretos-leyes; 22 de abril, que sirve para informar de Consejos Europeos, convalidar un Decreto-ley y celebrar una sesión de control al Gobierno; 6 de mayo, que aprueba las propuestas del Grupo Vasco, 20 de mayo y 3 de junio. Este último contará con 92 Diputados presentes en el hemiciclo, y se incorporarán además debates de totalidad sobre un convenio internacional y un proyecto de Ley.
} 
control sobre la actuación del Gobierno, sesiones de control que se seguirán celebrando de forma regular.

La única cuestión que podría ser de interés es plantear si debería haberse incrementado la presencia del Presidente del Gobierno en las Cortes Generales durante la pandemia, en vez de canalizar el control a través de las comparecencias del Ministro Illa en la Comisión de Sanidad y Consumo. Tal opción habría incrementado el control parlamentario sobre la actuación del Gobierno.

En todo caso, dentro de la vigencia del estado de alarma, el Gobierno impulsa unas nuevas medidas urgentes de prevención, contención y coordinación para hacer frente a la crisis sanitaria ocasionada por la covid-19 10 y establece un plan para la transición hacia una nueva normalidad el 28 de abril de $2020^{11}$. En este se definen y prevén una serie de fases $(0,1,2,3$ y nueva normalidad, sic). Aunque las Comunidades Autónomas pueden realizar solicitudes, es el Ministro de Sanidad el que decide la fase en que se encuentra cada Comunidad Autónoma. Y, una vez más, se han producido decisiones que, en ocasiones, han tenido más trasfondo político que sanitario ${ }^{12}$.

Sin embargo, los datos evolucionan negativamente y el Gobierno adopta un nuevo estado de alarma a través del citado Real Decreto 926/2020, de 25 de octubre, cuya vigencia se prorroga a través del Real Decreto 956/2020. Como es sabido, esta nueva declaración del estado de alarma aporta algunas novedades que han sido polémicas.

\footnotetext{
10 Real Decreto-ley 21/2020, de 9 de junio.

11 Este documento se acompaña de los Anexos I, II y III. Es relevante también la Orden SND/387/2020, de 3 de mayo, por la que se regula el proceso de cogobernanza con las comunidades autónomas y ciudades de Ceuta y Melilla para la transición a una nueva normalidad, que sitúa al Ministro de Sanidad como autoridad última para decidir (art. 6.3).

12 Nos remitimos, sin más trámite, a dos noticias de prensa: "El Gobierno dejó pasar de fase al País Vasco con más casos sospechosos que en Valencia". El Confidencial, 11 de mayo de 2020. Disponible en https:/www.elconfidencial.com/espana/comunidad-valenciana/2020-05-11/gobiernopais-vasco-valencia-sospechosos-coronavirus_2589251/, e "Illa se ve obligado a admitir que las decisiones sobre las desescalada solo las avaló con Simón". El Economista.es, 30 de julio de 2020, Disponible en https://www.eleconomista.es/economia/noticias/10698638/07/20/llla-se-veobligado-a-admitir-que-las-decisiones-sobre-las-desescalada-solo-las-avalo-Simon.html.
} 
fundación

Manuel Giménez Abad

deEstudios Parlamentarios ydel Estado Autonómico

La primera es que, aunque se señala como autoridad competente "el Gobierno de la Nación" (art. 2.1 in fine), se deja la adopción de decisiones a los Presidentes autonómicos, que actúan como autoridad competente delegada. Aunque el Defensor del Pueblo estima que tal posibilidad es compatible con la Constitución ${ }^{13}$, por estar prevista en el artículo 7 LOAES, existen relevantes razones para defender lo contrario. Señaladas, de forma sintética, serían las siguientes: (1) las autoridades delegadas no son tales, pues son ellas las que adoptan materialmente las restricciones de los derechos, ejerciendo así una competencia exclusiva del Gobierno de la Nación. Su papel no es ejecutivo, sino decisorio. Sostener lo contrario señalando que lo hacen en el marco del Real Decreto es lo mismo que afirmar que están sometidos al Estado de Derecho lo que, siendo obvio, es un problema distinto; (2) aceptar que las decisiones no las adopta el Gobierno de la Nación hace que la rendición de cuentas, prevista en el artículo 14 del Real Decreto, sea letra muerta. No tiene sentido que el Congreso cuestione al Ministro la decisión adoptada por un Presidente autonómico. Esta desconexión evidencia, por sí sola, el inadecuado uso que se ha hecho del estado de alarma, instrumento que dota de especiales poderes al Gobierno de la Nación, que este puede decidir ejercer, pero no delegar.

La segunda cuestión debatida es si se puede decretar la prórroga del estado de alarma por un periodo mayor de quince días (en concreto, del 9 de noviembre de 2020 al 9 de mayo de 2021). El Defensor del Pueblo entiende que sí, apoyándose en el precedente del Real Decreto 1717/2010, de 17 de diciembre, que prorrogó, con autorización del Congreso, el estado de alarma decretado por el Real Decreto 1673/2010, de 4 de diciembre, y en que no se impone esta previsión expresa ni en la Constitución ni en el Derecho español. Es una decisión libre del Congreso, se añadirá. Aunque estos argumentos no carecen de interés, existen también algunas razones que pueden ser invocadas en sentido contrario. La primera es general y abstracta, y es que el hecho de que exista un precedente que apoye esta

\footnotetext{
13 Ver, por todas, la resolución adoptada por el Defensor del Pueblo en relación con las solicitudes de recurso de inconstitucionalidad contra el Real Decreto 926/2020, la Resolución del 29 de octubre de 2020 del Congreso de los Diputados y el Real Decreto 956/2020, adoptada el 20 de enero de 2021, y disponible en https://www.defensordelpueblo.es/wpcontent/uploads/2021/01/Resolucion-compulsada-20027822_1.pdf.
} 
fundación

Manuel Giménez Abad

deEstudios Parlamentariosy del Estado Autonómico

conclusión no es un argumento de peso. La segunda es que hay sustanciales diferencias entre aquel supuesto y el que nos ocupa ahora que deben ser tomadas en consideración, y que son la grave incidencia del estado de alarma en los derechos fundamentales en todo el territorio estatal y que nos encontramos ahora ante una prórroga seis veces más amplia. ¿Resulta legítimo prorrogar una norma de estas características durante medio año? ¿Estamos en presencia de una medida proporcionada? Si se entiende que no, debería decretarse la nulidad de esta prórroga, sin que sirva como contraargumento que la decisión ha sido libremente adoptada por la Cámara Baja, porque esta se encuentra sometida, indiscutiblemente, a nuestra Constitución.

La tercera polémica que genera este Real Decreto es el modelo de rendición de cuentas previsto en su artículo 14, que se articula a través de una comparecencia del Presidente ante el Pleno cada dos meses, y de otra, mensual, del Ministro de Sanidad ante la Comisión parlamentaria de Sanidad y Consumo. Puede discutirse si tiene sentido que una medida inicialmente prevista para quince días pueda ser controlada de forma mensual. Pero este debate no llevaría muy lejos, ya que, con independencia de lo que diga esta norma, el Congreso conserva todos los mecanismos de control que la Constitución y el Reglamento de la Cámara le otorgan para fiscalizar la actuación del Gobierno. Si este control no es suficientemente intenso es porque no hay voluntad política de ejercerlo, y eso ya es cuestión distinta. Nos parece más grave, debe insistirse en ello, que tal control sea, en la práctica, inútil, puesto que las decisiones concretas se adoptan en las Comunidades Autónomas. Por esta razón, el debate político se traslada no a controlar estas, sino a discutir sobre si debe modificarse el Real Decreto para posibilitar la eventual adopción regional de medidas más o menos duras. Aunque esta cuestión es relevante, es distinta al control sobre la ejecución del estado de alarma. 


\section{LÍMITES DE LA CAPACIDAD DE LAS COMUNIDADES PARA INCIDIR SOBRE DERECHOS Y COMPETENCIAS}

En un Estado constitucional todos los órganos del Estado disponen de un poder limitado de actuación, que debe ejercerse en el marco del Estado de Derecho y con un escrupuloso respeto de los derechos fundamentales constitucionalmente consagrados.

Resulta evidente que el ejercicio de las competencias autonómicas puede incidir en el ejercicio de los derechos fundamentales ${ }^{14}$, y partiremos de esta premisa para examinar la materia arriba enunciada en el contexto de la pandemia.

La cuestión es si las Comunidades Autónomas pueden, en el contexto de un estado de alarma provocado por una crisis sanitaria, afectar ${ }^{15}$, por sí mismas, a los derechos fundamentales.

A nuestro entender, la incidencia autonómica sería limitada, en la medida en que su función sería que las Administraciones regionales (también las locales) ejecuten las decisiones adoptadas por el Gobierno de la Nación en el marco de sus competencias. Así ha sido durante el primer estado de alarma.

Sin embargo, como ya se ha anticipado, en el marco del segundo estado de alarma se ha nombrado a los Presidentes autonómicos autoridades competentes delegadas, y como tales, pueden "dictar, por delegación del Gobierno de la

\footnotetext{
14 Ver, en el plano doctrinal, por todos, Lucas Murillo de la Cueva, Enrique: "Delimitación de la competencia autonómica para la regulación de los derechos fundamentales". Revista de Derecho Político 46 (1989), pp. 11 y ss. (esp. p. 37).

${ }^{15}$ Se utiliza esta expresión porque, como es sabido, la teoría indica que el estado de alarma no puede conllevar la suspensión de los derechos fundamentales. Optamos, así, por no entrar en una polémica que ha generado un intenso debate doctrinal. Ver, entre otros, Velasco Caballero, Francisco "¿Estamos ante una "suspensión" de derechos fundamentales? Más bien no", disponible en https://franciscovelascocaballeroblog.wordpress.com/2020/04/05/estamos-ante-una-suspensionde-derechos-fundamentales-mas-bien-no/; Álvarez García, Francisco Javier: "Estado de alarma o de excepción". Estudios Penales y Criminológicos XL (2020), pp. 1 y ss., disponible en https://editorial.tirant.com/free_ebooks/E000020005117.pdf; y Leiva Escudero, Guillermo: "Constitucionalidad de las restricciones a la libertad de circulación en el estado de alarma por el coronavirus Covid-19". Diario La Ley 11/05/2020, disponible en https://diariolaley.laleynext.es/dll/2020/05/20/constitucionalidad-de-las-restricciones-a-la-libertadde-circulacion-en-el-estado-de-alarma-por-el-coronavirus-covid-19.
} 
fundación

Manuel Giménez Abad

deEstudios Parlamentarios y del Estado Autonómico

Nación, las órdenes, resoluciones y disposiciones para la aplicación de lo previsto en los artículos 5 a 11. Para ello, no será precisa la tramitación de procedimiento administrativo alguno ni será de aplicación lo previsto en el segundo párrafo del artículo 8.6 y en el artículo 10.8 de la Ley 29/1998, de 13 de julio, reguladora de la Jurisdicción Contencioso-administrativa". Este último inciso supone que no resultará preciso que la autoridad judicial autorice o ratifique dichas medidas.

Ya hemos expresado que esta nueva formulación del estado de alarma contraviene, a nuestro modesto entender, el texto constitucional, porque es un mecanismo que permite la adopción de decisiones al Gobierno de la Nación, siendo esta competencia indelegable. Ahora debemos añadir que es una norma sorprendente, porque confiere un poder individual al Presidente autonómico. En puridad, es competencia suya, no del Gobierno regional, dictar las medidas que estime oportunas en el marco, obviamente, del Real Decreto que establece el estado de alarma. $Y$ este es el primero de los problemas que suscita este estado de alarma.

Otro tema interesante es determinar si las decisiones adoptadas por un Presidente autonómico pueden ser posteriormente modificadas por una decisión del Gobierno de la Nación. Se afirma, en este sentido ${ }^{16}$, que el último responsable del estado de alarma es el Gobierno central y que todas las autoridades deben obedecer sus indicaciones incluso en estas circunstancias (art. 10.3 LO 4/1981), por lo que el Gobierno podría actuar en contra de lo previsto por un responsable regional. Pero el asunto no es tan evidente, dado que el Gobierno, libremente, y con autorización del Congreso, ha configurado un marco general que permite la actuación de los Presidentes autonómicos. Si desea restringir esta actuación debería modificar el Real Decreto que está en vigor o, en el caso de que una Comunidad actúe fuera del marco del estado de alarma, deberá impugnar su actuación.

En todo caso, y en tercer lugar, esta novedosa (y discutible) construcción del estado de alarma se ha justificado en la cogobernanza. Ya se ha señalado que la misma no cabe cuando la Constitución atribuye, de forma excepcional, un poder

\footnotetext{
16 Ver Esteban, Rocío: “¿Todo el poder para el presidente?”, La Razón, 26 de octubre de 2020, disponible en https://www.larazon.es/espana/20201026/peklvftb6nev3kcpt6azbc7xkm.html.
} 
acrecido al Gobierno de la Nación, salvo en la fase ascendente de la decisión (esto es, en la propia conformación de las medidas que el Gobierno adopta). Y ahora debemos añadir que tal gobernanza no ha existido realmente, salvo que se identifique esta con un poder descentralizado (parecido al que opera entre las bases y la legislación de desarrollo), y no, como parece indicar el sentido literal de la expresión, en un gobierno compartido.

Es verdad que la descentralización de las decisiones relacionadas con la pandemia ha sido (torpemente) solicitada por algunas Comunidades Autónomas ${ }^{17}$, y facilitada por el Gobierno de la Nación, que les transfiere así el coste político de las restricciones adoptadas. Esto ha generado también, diecisiete centros de decisión que han ido modificando sus decisiones de un día para otro, lo que no resulta tampoco beneficioso para el principio de seguridad jurídica.

Por otra parte, cuando las cosas se han puesto realmente complicadas y algunas Comunidades han solicitado que se reforme el catálogo de restricciones posibles y se incorporen otras más gravosas (adelantamiento del toque de queda a las 18:00 o 20:00 horas, confinamiento domiciliario selectivo de municipios o zonas de salud $^{18}$ ), el Gobierno de la Nación se ha negado (no tanto, a nuestro juicio, por razones de salud pública, como por no retrasar las elecciones autonómicas catalanas y tener que someterse, nuevamente, al control parlamentario).

En todo caso, se ha producido un hecho más que debe ser brevemente glosado. Y es que la Junta de Castilla y León ha decidido adelantar el toque de queda a las 20:00 horas ${ }^{19}$, cuando lo previsto en el artículo 5.2 del Real Decreto 926/2020,

17 "Hasta diez comunidades autónomas reclaman a Sánchez que declare el estado de alarma y ceda su gestión", Telemadrid, 23 de octubre de 2020, disponible en https://www.telemadrid.es/coronavirus-covid-19/comunidades-autonomas-reclaman-Sanchezdeclare-2-2280092022--20201023103430.html.

18 "Cuatro comunidades piden al Gobierno que autorice el confinamiento domiciliario". El País, 14 de enero de 2021, disponible en https://elpais.com/sociedad/2021-01-14/cuatro-comunidadespiden-al-gobierno-que-autorice-el-confinamiento-domiciliario.html; y "Casi todas las autonomías pedirán al Gobierno cambiar el estado de alarma para adelantar el toque de queda", El Periódico, 20 de enero de 2021, disponible en

https://www.elperiodico.com/es/sociedad/20210120/autonomias-pediran-gobierno-modificaralarma-11466105.

${ }^{19}$ Acuerdo 2/2021, de 15 de enero, del Presidente de la Junta de Castilla y León, como autoridad competente delegada dispuesta por el Real Decreto 926/2020, de 25 de octubre, por el que se 
modificado, es que "la autoridad competente delegada correspondiente podrá determinar, en su ámbito territorial, que la hora de comienzo de la limitación prevista en este artículo sea entre las 22:00 y las 00:00 horas y la hora de finalización de dicha limitación sea entre las 5:00 y las 7:00 horas".

Que tal adelanto es contrario a Derecho es fácil de acreditar. De hecho, la propia Comunidad Autónoma había interesado el adelanto de la hora de toque posible al Gobierno de la Nación y, como sabemos, este se había negado. Además de contar con una norma clara, y que debe ser interpretada de forma restrictiva por afectar al ejercicio de derechos fundamentales, la Junta ha defendido la legitimidad de su medida en el artículo 10 de la misma norma, que permite "modular, flexibilizar y suspender la aplicación de las medidas previstas en los artículos 5, 6, 7 y 8, con el alcance y ámbito territorial que determine". Es evidente que este argumento es desafortunado, puesto que ese precepto lleva por revelador título "Flexibilización y suspensión de las limitaciones", y lo que se ha hecho es justamente lo contrario ${ }^{20}$.

El propio Gobierno de la Nación se ha visto forzado a impugnar la decisión autonómica, por ser incompatible con el Real Decreto que regula el estado de alarma. Sorprendentemente, tratándose de una norma que restringe seriamente derechos fundamentales, no ha pedido la adopción de medidas cautelarísimas ${ }^{21}$,

declara el estado de alarma para contener la propagación de infecciones causadas por la SARSCoV-2, por el que se determinan las horas de comienzo y finalización de la limitación de la libertad de circulación de las personas en horario nocturno, disponible en http://bocyl.jcyl.es/html/2021/01/16/html/BOCYL-D-16012021-1.do.

20 Todos estos argumentos se explican adecuadamente en el artículo de Camino Vidal Fueyo, "La covid es letal, pero seguimos en un Estado de Derecho", El Norte de Castilla, 16 de enero de 2021, disponible en https://www.elnortedecastilla.es/opinion/covid-letal-seguimos-20210116184128nt.html. Manuel Izquierdo Carrasco estima, por su parte, que el artículo 10 del Real Decreto permite adoptar mayores restricciones, siempre que afecten al "horario nocturno", se comunique la medida al Ministerio de Sanidad y se respeten los acuerdos del Consejo Interterritorial del Sistema Nacional de Salud, como aquí ocurre. Esta tesis se sostiene en "20.00 horas un cierre con base legal ya", El Mundo, 19 de enero de 2021, disponible en

https://www.iustel.com/diario_del_derecho/noticia.asp?ref_iustel=1207067. Entendemos que esta interpretación resulta incompatible con la delimitación horaria fijada en el artículo 5 y supone realizar una interpretación extensiva en la restricción de derechos, incompatible con nuestra Constitución.

${ }^{21}$ Ramos, F: "El Gobierno se rinde a que el toque de queda en Castilla y León siga en vigor desde las ocho de la tarde". Diario de Castilla y León, 18 de enero de 2021, disponible en 
sino cautelares ${ }^{22}$, por lo que se ha seguido aplicando hasta que el Tribunal Supremo se ha pronunciado sobre éstas, suspendiendo el adelanto del toque de queda ${ }^{23}$.

Lo grave de la decisión del Presidente de la Comunidad Autónoma de Castilla y León es que estamos en presencia de un desplante manifiesto al Estado de Derecho, y ante una muestra evidente de ausencia de lealtad institucional, que es uno de los males endémicos que sufre nuestro Estado Constitucional. Y esta opinión no se puede ver alterada ni porque la finalidad de la medida sea positiva ni porque sea criticable, que lo es, que el Gobierno de la Nación se inhiba ante el recrudecimiento, evidente, de la pandemia.

En resumen, la activación del estado de alarma hace que sea el Gobierno de la Nación el responsable de adoptar las medidas restrictivas de derechos que pretendan atajar la crisis sanitaria, sin que ello impida a las Comunidades Autónomas actuar en el marco de sus competencias (restringiendo, por ejemplo, los horarios comerciales). Partiendo de esta premisa, debe concluirse que el modelo de estado de alarma por el que se ha optado, y la posterior actuación autonómica no se han revelado eficientes en el plano sanitario.

https://diariodecastillayleon.elmundo.es/articulo/castilla-y-leon/gobierno-rinde-toque-queda-castillaleon-siga-vigor-tarde/20210118130238022373.html.

22 EI Periódico: "El Supremo da 10 días a Castilla y León para defender el toque de queda a las 20 horas". El Periódico, 19 de enero de 2021, disponible en

https://www.elperiodico.com/es/politica/20210119/supremo-recurso-gobierno-toque-queda-castillay-leon-11464044.

${ }^{23}$ A través del ATS (Sala de lo Contencioso-Administrativo, Sección 4) 1156/2021, de 16 de febrero (ECLI:ES:TS:2021:1156A, disponible

en https://www.poderjudicial.es/search/AN/openDocument/62eba54d70c96604/20210217). El Tribunal Supremo no ha evacuado su resolución sobre estas medidas cautelares tan pronto como podía hacerlo, lo que resulta también muy preocupante cuando tal decisión se inscribe en el marco de la adopción de un estado de alarma y se supone que resulta esencial que los controles (políticos y, como es el caso, jurídicos) se ejerzan con especial prontitud en estos casos. 
fundación

Manuel Giménez Abad

de Estudios Parlamentarios ydel Estado Autonómico

\section{VALORACIÓN DE LAS RELACIONES INTERGUBERNAMENTALES DURANTE LA PANDEMIA}

No sorprenderá al amable lector que haya llegado hasta aquí que nuestra valoración sea también negativa en este punto. Ya se han realizado consideraciones previas que abonan esta conclusión, y que ahora nos limitamos a presentar de forma cronológica.

El primer estado de alarma ha supuesto que el Gobierno de la Nación adoptara severas restricciones a la movilidad que fueron, en un primer momento, entendidas por todas las Comunidades Autónomas. Sin embargo, a medida que el confinamiento se prolongaba, algunas Comunidades Autónomas, especialmente la catalana y la vasca, han ido exigiendo un mayor protagonismo (primero en la fallida desescalada y después en el marco del nuevo estado de alarma). Dado que sus votos han sido necesarios en el Congreso de los Diputados, el Gobierno de la Nación ha cedido a estas peticiones.

Ahora bien, como es bien sabido, esto no ha ocurrido en relación con la Comunidad Autónoma de Madrid. Las tensiones han sido permanentes a partir de la desescalada, en la que el paso de fase ha sido más lento que en otras Comunidades Autónomas que tenían peores datos, especialmente a raíz de la celebración del Consejo Interterritorial del Sistema Nacional de Salud celebrado el 30 de septiembre de 2020. Como se recordará, el Ministro propuso la adopción de una serie de medidas restrictivas que afectaban a varios municipios de la Comunidad de Madrid, medidas que son rechazadas por algunas Comunidades Autónomas. Pese a ello, el Gobierno decide imponerlas a través de una ambigua resolución de 30 de septiembre de 2020 de la Secretaría de Estado de Sanidad, por la que se da publicidad al citado Acuerdo ${ }^{24}$. Se trata de una decisión que plantea algunos problemas jurídicos. En primer lugar, porque, de acuerdo con lo previsto en el artículo 73.2 de la Ley $16 / 2003$, de 28 de mayo, de cohesión y calidad del Sistema Nacional de Salud, "los acuerdos del Consejo se plasmarán a

24 Publicada en el BOE de 1 de octubre de 2020, y disponible en https://www.boe.es/boe/dias/2020/10/01/pdfs/BOE-A-2020-11590.pdf.

16

Palacio de la Aljafería - Calle de los Diputados, s/n- 50004 ZARAGOZA Teléfono 976289715 fundacion@fundacionmgimenezabad.es - www.fundacionmgimenezabad.es 
través de recomendaciones que se aprobarán, en su caso, por consenso"25. Esto es, no son válidos si no hay consenso, y además no tienen fuerza jurídica vinculante ${ }^{26}$. Para evitar estos evidentes problemas, e imponer su voluntad, el Ministro se apoya, en la citada Resolución, en dos preceptos legales.

El primero es el artículo 65.1 de la citada Ley 16/2003, que dispone que "la declaración de actuaciones coordinadas en salud pública corresponderá al Ministerio de Sanidad, previo acuerdo del Consejo Interterritorial del Sistema Nacional de Salud, con audiencia de las Comunidades directamente afectadas, salvo en situaciones de urgente necesidad, en cuyo caso se tomarán las medidas que sean estrictamente necesarias y se le informará de manera urgente de las medidas adoptadas". Dado que no existió "acuerdo" en el Consejo Interterritorial debe entenderse que las medidas se adoptan al amparo de la existencia de una urgente necesidad.

El segundo es el artículo 151.2.a) de la Ley 40/2015, de 1 de octubre, de Régimen Jurídico del Sector Público27, que dispone, en lo que ahora interesa, que, "cuando la Administración General del Estado ejerza funciones de coordinación, de acuerdo con el orden constitucional de distribución de competencias del ámbito material respectivo, el Acuerdo que se adopte en la Conferencia Sectorial, y en el que se incluirán los votos particulares que se hayan formulado, será de obligado cumplimiento para todas las Administraciones Públicas integrantes de la Conferencia Sectorial, con independencia del sentido de su voto". Lo que la Abogacía del Estado defiende es que este precepto legal ha derogado el artículo 73.2 de la Ley 16/2003, ya que, siempre a su parecer, el Consejo Interterritorial es

\footnotetext{
25 La ley está disponible en https://www.boe.es/buscar/act.php?id=BOE-A-2003-10715. En el mismo sentido, el artículo 14 del Reglamento establece que los acuerdos se pueden canalizar a través de recomendaciones o convenios, cuando estemos en presencia de acuerdos de cooperación para llevar a cabo acciones sanitarias comunes, cuestión distinta a la aquí tratada. El reglamento puede consultarse en https://www.mscbs.gob.es/organizacion/consejolnterterri/docs/ReglamentoCISNS-23-07-2003.pdf. ${ }^{26}$ Afirmación esta que puede combatirse invocando el artículo 151 de la Ley 40/2015, de régimen jurídico del sector público, que regula esta cuestión para todas las Conferencias Sectoriales (como el CISN). Esta ley señala que los acuerdos obligarán a todas las Comunidades Autónomas, aunque voten en contra, si el Estado ejerce competencias de coordinación, como es el caso.

27 Disponible en https://www.boe.es/buscar/act.php?id=BOE-A-2015-10566.
} 
una Conferencia Sectorial ${ }^{28}$, aunque curiosamente, poco tiempo antes había defendido ante el Tribunal Supremo que se debía inadmitir un acuerdo del propio Consejo por entender que se impugna una (mera) recomendación ${ }^{29}$. Aunque resulta posible entender que el Consejo Interterritorial opera como una Conferencia Sectorial ${ }^{30}$, resulta más complicado negar que el artículo 73.2 de la Ley 16/2003 (que cita el Tribunal Supremo) pueda considerarse una norma especial $^{31}$ frente a la general prevista en la Ley 40/2015.

En todo caso, resulta suficiente, a nuestro juicio, el título previsto en el artículo 65.1 de la Ley 16/2003 para que el Ministro de Sanidad pueda adoptar, por sí mismo y con independencia de lo acaecido en el Consejo Interterritorial, medidas sanitarias.

Cuestión distinta, claro, es que estas medidas puedan comprometer el ejercicio de derechos fundamentales. Lo que va a ocurrir es que, una vez que la Comunidad de Madrid decide, lealmente, aplicar unas medidas sanitarias en las que no $\mathrm{cree}^{32}$, estas no van a ser ratificadas por la autoridad judicial por restringir derechos fundamentales ${ }^{33}$. Considera el Tribunal que el artículo 65 de la Ley 16/2003 no

${ }^{28}$ Esta información se obtiene a través de la prensa (ver, por ejemplo y por todos, El País, 30 de septiembre de 2020, disponible en: https://elpais.com/sociedad/2020-09-30/las-decisiones-delconsejo-territorial-de-sanidad-por-consenso-o-por-decision-colegiada.html).

29 ATS (Sala 3, Sección 4ª) 7240/2020, de 30/09/2020, en recurso 257/2020 (ECLI:ES:TS:2020:7240A), Razonamiento Jurídico 2 in fine. El Auto está disponible en https://www.poderjudicial.es/search/AN/openDocument/721413f09ac80686/20201002.

30 Como hace el Tribunal Supremo en el Auto que se acaba de citar, a fin de determinar que sus acuerdos no pueden imputarse al Gobierno, y que la competencia judicial para pronunciarse sobre su validez corresponde, entonces, a la Audiencia Nacional (ibídem, razonamiento jurídico 5).

${ }^{31}$ Como sostiene Vicente Lomas Hernández en "Naturaleza jurídica del Consejo Interterritorial del Sistema Nacional de Salud". El Derecho, 22 de octubre de 2020, disponible en https://elderecho.com/naturaleza-juridica-del-consejo-interterritorial-del-sistema-nacional-de-salud.

32 Orden 1273/2020, de 1 de octubre, de la Consejería de Sanidad, por la que se establecen medidas preventivas en determinados municipios de la Comunidad de Madrid en ejecución de la Orden del Ministro de Sanidad, de 30 de septiembre de 2020, por la que se aprueban actuaciones coordinadas en salud pública.

${ }^{33}$ ATSJ Madrid (Sala de lo Contencioso-Administrativo, Sección 8a ) 128/2020, de 8 de octubre, procedimiento 1224/2020, disponible en http://milansabogados.com/wpcontent/uploads/2020/10/Auto-TSJM-Sala-Contencioso-Advo.-Sec.-8\%C2\%AA-8-oct-2020.pdf, que fue ratificado en reposición por Auto del mismo Tribunal 357/2020, de 29 de octubre (ECLI: ES:TSJM:2020:357A), disponible en

https://www.poderjudicial.es/search/AN/openDocument/4571d7f0ea32f06e/20201102. En esta última resolución es muy recomendable la lectura del FJ 5, que niega que los poderes estatales de coordinación puedan desconocer los títulos competenciales que posee la Comunidad Autónoma. 
contiene una habilitación legal para establecer medidas limitativas del derecho fundamental a la libre circulación (FJ 6). Y esto explica que el Gobierno opte por una nueva declaración del estado de alarma circunscrita, en este caso, a una serie de municipios de la Comunidad de Madrid, a través del Real Decreto 900/2020, de 9 de octubre ${ }^{34}$, imponiendo su criterio al de la Comunidad de Madrid. Aunque puede someterse a debate si las motivaciones del Gobierno no son solamente sanitarias, sino también políticas, y si resultaba imprescindible (léase proporcionado) declarar en este supuesto un estado de alarma ${ }^{35}$, lo que interesa subrayar es que no ha habido coordinación intergubernamental sino imposición del Gobierno estatal.

Una buena prueba de esta afirmación la encontramos en lo que estamos viviendo ahora. Según el Real Decreto que declara el estado de alarma para determinados municipios que forman parte de la Comunidad de Madrid, "una tasa de incidencia superior a los 500 casos por 100.000 habitantes, que duplica la considerada de alto riesgo, permite establecer un umbral significativamente elevado a partir del cual puede calificarse la situación como extrema, y da perfecta cuenta de la enorme gravedad de la propagación de la enfermedad en las unidades territoriales que la experimentan. A partir de este umbral, resulta imprescindible la adopción con urgencia de medidas de choque dirigidas a tratar de controlar en el menor tiempo posible la situación extrema generada". Pues bien, en fechas recientes, como ya se ha indicado, un número significativo de Comunidades Autónomas han pedido al Gobierno que modifique el actual estado de alarma permitiendo que los Presidentes autonómicos puedan adelantar el toque de queda o decretar confinamientos domiciliarios. Estas medidas se han solicitado con tasas de contagios mucho más elevadas ${ }^{36} \mathrm{y}$, sin embargo, el Gobierno de la Nación se ha

\footnotetext{
${ }^{34}$ Ver, en el plano doctrinal, Berzosa López, Daniel: "Análisis de urgencia del estado de alarma para la Comunidad de Madrid". Diario La Ley 9720, 2020; y Fernández de Gatta Sánchez, Dionisio: "El Real Decreto 900/2020, de 9 de octubre: nuevamente se declara el estado de alarma (pero...únicamente sobre Madrid)", Diario La Ley 9720, 2020.

${ }^{35}$ Los dos autores citados en la nota anterior se plantean la primera cuestión, y el citado en último lugar la segunda.

${ }^{36}$ Ver la nota 18 del presente trabajo y, en relación con esto, y como ejemplo, los datos del 15 de enero de 2021 que se aportan por el propio Ministerio, y disponibles en https://www.mscbs.gob.es/profesionales/saludPublica/ccayes/alertasActual/nCov/documentos/Actu
} 
negado, por considerarlas innecesarias. Más allá de que intereses políticos (como puede ser la próxima celebración de las elecciones catalanas) nublen el criterio del ejecutivo estatal, resulta evidente que estamos ante comportamientos incongruentes.

Cuestionar la inacción del Gobierno de la Nación, que ha hecho recaer todo el peso de la lucha contra la pandemia en las Comunidades Autónomas, no legitima, claro está, que estas actúen al margen del Estado de Derecho. Esto es lo que ha hecho, a nuestro parecer, la Junta de Castilla y León, imponiendo un toque de queda a las 20:00 horas que es incompatible con el artículo 5.2 del Real Decreto 926/2020. No resulta preciso insistir en nuestra valoración, que ya se ha justificado en líneas anteriores.

\section{LECTURAS PARA EL FUTURO}

La crisis sanitaria que hemos vivido evidencia, a nuestro parecer, algunas virtudes y defectos de nuestro modelo constitucional que merece la pena glosar brevemente.

El primer acierto es el modelo constitucional del estado de alarma, rectamente entendido. Creo que nadie discute seriamente que habilitar al Gobierno de la Nación para que pudiera adoptar determinadas decisiones, más allá del acierto o desacierto de estas, era absolutamente necesario para tratar de atajar la pandemia. Tiene sentido, además, que todos los medios técnicos y humanos se pongan a disposición de una misma autoridad, que debe actuar en aras del interés general. No parece absurdo que, en una situación como la descrita, se haga un uso centralizado de las camas $\mathrm{UCl}$, o se realice una compra unificada de material sanitario (como la propia Unión Europea ha hecho con las vacunas).

El segundo acierto es el correcto funcionamiento del Estado de Derecho, que ha permitido someter a control judicial la actuación desarrollada, en el marco de la

alizacion_291_COVID-19.pdf. Para tener una visión de la evolución resulta útil consultar https://www.porcentual.es/coronavirus-mapa/. 
fundación

Manuel Giménez Abad

deEstudios Parlamentarios ydelEstado Autonómico

crisis sanitaria, por los poderes públicos. Las decisiones del Gobierno de la Nación y de las Administraciones territoriales solamente son admisibles si respetan nuestro ordenamiento constitucional (en especial, el reparto competencial y los derechos fundamentales).

Lo ocurrido en nuestro país también ha servido para evidenciar las deficiencias del modelo autonómico del Estado, la escasa capacidad de las Administraciones para coordinar sus actuaciones, y la falta de lealtad institucional entre el Gobierno y las Administraciones territoriales, que tensiona el Estado de Derecho. Lo más sorprendente es que no se haya aprovechado el primer estado de alarma (plenamente justificado) para elaborar un marco legislativo que pudiera mostrarse útil para afrontar futuras crisis sanitarias (por ejemplo, a través de la elaboración de una Ley de pandemias) o buscar, de verdad, mecanismos eficaces de coordinación interadministrativa. Son, a fecha de hoy, tareas pendientes. 